\title{
Millimetre-Wave MIMO Array of a Compact Grid Antenna for 5G Wireless Networks and Beyond
}

\author{
Syeda Fizzah Jilani ${ }^{1,2}$, Qammer H. Abassi ${ }^{3}$, and Akram Alomainy ${ }^{2}$ \\ ${ }^{1}$ Frontier Institute for Research in Sensor Technologies (FIRST), University of Maine, Orono 04469 ,USA \\ ${ }^{2}$ School of Electronic Engineering and Computer Science, Queen Mary University of London, London, E1 4NS, UK \\ ${ }^{3}$ James Watt School of Engineering, University of Glasgow, Glasgow G12 8QQ, UK \\ \{s.f.jilani, a.alomainy\}@qmul.ac.uk
}

\begin{abstract}
This paper presents a 4-element multiple-inputmultiple-output (MIMO) configuration of planar and compact two-dimensional (2D) grid antennas. The proposed grid is a 2D array of thirteen circular patches each with a radius of $0.175 \lambda_{0}$, having a single feed point. Measured results of the singleelement microstrip-fed grid-array antenna show a $6 \mathrm{GHz}$ bandwidth in the range of $27-33 \mathrm{GHz}$. The realized gain is above $9 \mathrm{dBi}$ in almost the whole range with a peak gain of $11.57 \mathrm{dBi}$ at $29 \mathrm{GHz}$. The numerically calculated antenna efficiency is above $80 \%$. The transmission characteristics of the 4-element MIMO array are below $-27 \mathrm{~dB}$ that indicates low mutual coupling and high isolation. These high-performance aspects of the proposed millimetre-wave (mm-wave) MIMO antenna array validate its potential for the $5^{\text {th }}$ generation (5G) cellular devices especially in indoor base-stations.
\end{abstract}

Keywords-5G, antennas, array, millimeter-wave, MIMO.

\section{INTRODUCTION}

The recent advancement in wireless networks towards $5 \mathrm{G}$ and beyond necessitate the utilisation of unused resources of bandwidth at mm-wave spectrum. Higher bandwidth ensures a high-speed communication while sparing enough room for the future upgrades $[1,2]$. Current $5 \mathrm{G}$ wireless networks have initiated utilisation of the $28 \mathrm{GHz}$ band to mitigate bandwidth scarcity issues $[3,4]$. This spectrum transition towards mmwaves is anticipated to deliver numerous advantages such as; shorter wavelengths for smaller antenna form factor, channelwidth enhancement, wideband spread-spectrum ability, as well as an accessibility to certain high-attenuation bands for a secure and short-distance point-to-point link $[5,6]$. The $5 \mathrm{G}$ architecture is highly reliant on versatile antenna designs to deal with the critical challenges of the mm-waves spectrum. One major challenge is to overcome the high free-space path loss, which severely attenuates the signal strength through the blockages or long transmission distances [7]. In order to resolve this issue, an ultra-dense and short-range cell area is recommended where the advanced indoor base-stations with high-performance antennas will be used. Compact MIMO antenna arrays offer a smart choice to fulfil the high-gain demands required for mm-wave path loss compensation and enhanced bandwidth for high data rates. Additionally, a MIMO array comprising of multiple wideband antennas is suggested as essential for future communication networks, to ensure higher capacity, capability to mitigate multipath fading effects, improvement of the transmission quality, better coverage, and many other attractive features [8].

Antennas being the centralised unit of any wireless system have recently gathered huge attention and several novel design techniques have been established to accomplish exceptional performance. Conventional geometries of patch antennas though offer planar integration and ease of fabrication and installation, still suffer from limited gain and bandwidth. Bandwidth can be increased with structural alterations, such as addition of slots, fractals and monopole geometries, which are often associated with lower gain and efficiency [9]. On the other hand, antenna gain can be improved with a larger radiating area by using parasitic patches or arrays, which typically compromise on compactness due to a significant extend in the size and holds limited bandwidth [10]. The antenna elements of arrays are spaced apart to avoid mutual coupling and formation of grating lobes and are designed with a well-matched feeding network thus causing an additional increase in the overall area of a prototype.

Recent developments on antennas for $5 \mathrm{G}$ and beyond have addressed these challenges, efforts have been set forth to deal with these shortcomings and emphasized on simultaneously achieving high gain and wide bandwidth [11-13]. Several linear and 2D grid antenna arrays have been proposed for $5 \mathrm{G}$ applications with high gain and wider bandwidth while preserving the compactness [13-16]. This paper presents a versatile MIMO antenna array to combine the advantages of high gain, wide bandwidth, high diversity and compact structure. A planar and compact 2D grid of circular radiating patches is designed as a single antenna at $5 \mathrm{G}$ band of $28-\mathrm{GHz}$ and utilised in a 4-element MIMO assembly.

\section{MIMO ANTENNA DESIGN AND ANALYSIS}

\section{A. Single Antenna Element}

The grid geometry of the antenna is designed with thirteen circular patches each with a radius of $0.175 \lambda_{0}$ (where wavelength, $\lambda_{o}=$ speed of light $(c) /$ resonant frequency $\left(f_{o}\right)$, estimated at resonant/centre frequency $\left.\left(\mathrm{f}_{\mathrm{o}}\right)=30 \mathrm{GHz}\right)$. Rogers $\mathrm{RT} /$ Duroid $5880\left(\varepsilon_{\mathrm{r}}=2.2\right.$, and $\left.\tan \delta=0.0009\right)$ of $21.5\left(2.15 \lambda_{\mathrm{o}}\right)$ $\times 23\left(2.3 \lambda_{0}\right) \times 0.8\left(0.08 \lambda_{0}\right) \mathrm{mm}^{3}$ dimensions is used as a substrate material. The design contains a continuous bottom copper ground. The top surface of the substrate includes the designed grid along with a well-matched network of feedlines that connects the radiating patches, symmetrically organised in a planar 2D grid structure [16]. Microstrip feed line with a stub of optimised dimensions is designed for the impedance matching between the single feed point and the grid.

The simulation modelling of the proposed antenna as well as the computational analysis of the electromagnetic (EM) performance are performed in the CST STUDIO SUITE software. The $50-\Omega$ matched $\mathrm{K}$-connector is also designed as a part of the simulated antenna model to visualise the close approximation of the real-time measurement process. Fig. 1 shows the designed antenna grid array with the optimised dimensions obtained after careful a parametric analysis. The fabrication of the designed antenna array is carried out by using the in-house LPKF milling machine. Rogers RT/Duroid substrate with a copper cladding thickness of $17.5 \mu \mathrm{m}$ on both sides of the substrate is used. The prototyping is done on one side of the substrate while the bottom metal cladding remained intact as a continuous ground plane. 


\section{University of Glasgow}

Jilani, S. F., Abbasi, Q. H. and Alomainy, A. (2020) Millimetre-Wave MIMO Array of a Compact Grid Antenna for 5G Wireless Networks and Beyond. In: 5th International Conference on the UK-China Emerging Technologies (UCET 2020), Glasgow, UK, 20-21 Aug 2020, ISBN 9781728194882 (doi:10.1109/UCET51115.2020.9205326).

This is the author's final accepted version.

There may be differences between this version and the published version. You are advised to consult the publisher's version if you wish to cite from it.

\section{http://eprints.gla.ac.uk/221494/}

Deposited on: 27 July 2020

Enlighten - Research publications by members of the University of Glasgow http://eprints.gla.ac.uk 


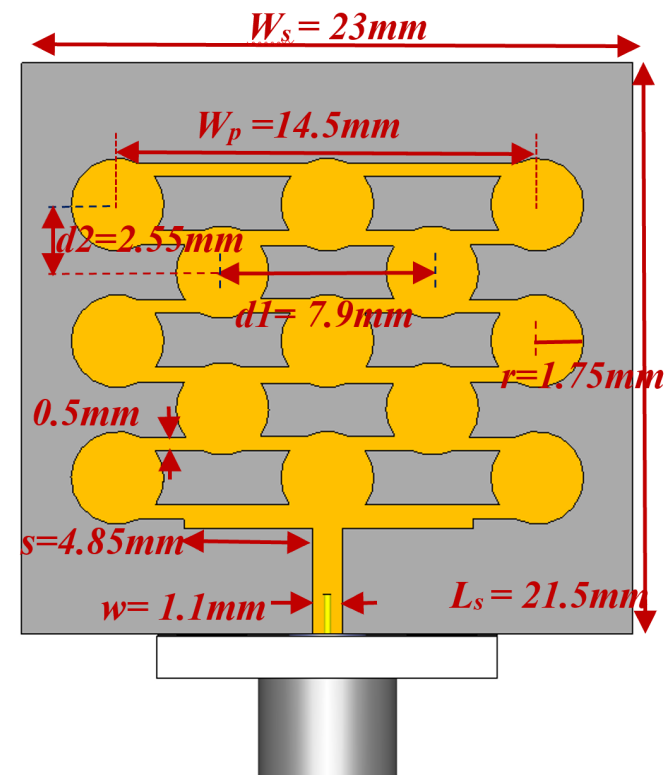

Fig. 1. Proposed compact grid antenna element (single radiator) with parametrically optimised dimensions.

\section{B. 4-Element MIMO Array}

The next step is to incorporate the antenna design into a four-element MIMO configuration for cellular base-station units, as well as in printed circuit board (PCB) layouts for wireless devices. The placement of all the four antennas has been carried out on a single substrate of $42 \times 42 \times 0.8 \mathrm{~mm}^{3}$. The conventional array design requires that minimum spacing between the patches should be $\lambda / 2$ or more (i.e. to minimise coupling effects). Here, as each grid antenna itself is an array of multiple circular patches, thus the minimum spacing criteria was justified with a $7.9 \mathrm{~mm}$ distance between the centres $(d 1)$ (as $\lambda_{o}=10 \mathrm{~mm}$ at $f_{o}=30 \mathrm{GHz}$ ). In order to lower the mutual coupling between the individual grid antennas and increase the individual independence, an orthogonal arrangement is suggested where each antenna is placed orthogonally to the adjacent antenna. Four K-connectors for individual antennas are also included in the simulated MIMO design for accurate modelling. Fig. 2 shows the simulated model of the proposed 4-element MIMO antenna array.

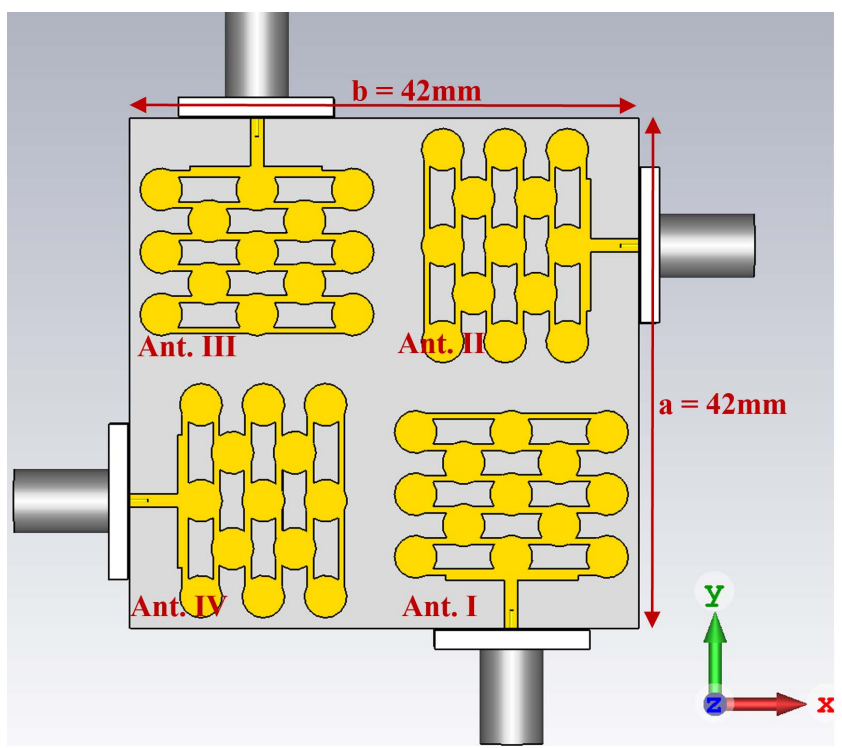

Fig. 2. Proposed compact grid 4-element MIMO antenna array with parametrically optimised dimensions.

\section{RESULTS AND DISCUSSION}

The performance of the compact grid MIMO antenna array is fully analysed in the computational parametric analysis in CST software. The S-parameters, radiation patterns, surface current density and realised gain have been examined in order to evaluate the proposed antenna performance.

\section{A. Single Antenna Element}

The fabricated single element grid antenna prototype is shown in the inset figure of Fig. 3. Experimental tests of the fabricated prototype by using the Vector Network Analyser (VNA) and a near-field scanning setup validate the simulation outcomes. Fig. 3 shows the reflection coefficient $\left(S_{11}\right)$ plots with simulated bandwidth of $27.5-33.3 \mathrm{GHz}$ with $-10 \mathrm{~dB}$ reference, while the measured results cover a range of 27-33 $\mathrm{GHz}$. This refers to a good agreement between the two plots, though slight shift towards lower frequency is observed in the measured profile may be due to the fabrication intolerances or because of some insignificant measurement discrepancies from connector/cables losses.

The simulated and measured normalised radiation patterns of the proposed grid antenna at $\mathrm{E}$ and H-plane cuts are shown in Fig. 4. The E-plane plots show that the antenna radiation splits into two beams towards broadside and the side lobes are below $-10 \mathrm{~dB}$ as in H-plane plots. Table I shows the realised gain of the proposed antenna at distinct frequencies of the operating range. The simulation results (referred as sim. in Table I) showed that a realised gain of above $9 \mathrm{dBi}$ is observed in $27.5-33 \mathrm{GHz}$ range, with a peak gain of $11.31 \mathrm{dBi}$ at 29 GHz. Measurements (i.e. Mea. in Table I) of the fabricated antenna show close results with a peak gain of $11.57 \mathrm{dBi}$, though profile observes same shift towards lower frequency as in the $S_{11}$ plot. The total antenna efficiency obtained from the CST computation is $80 \%$ or above in the entire bandwidth.

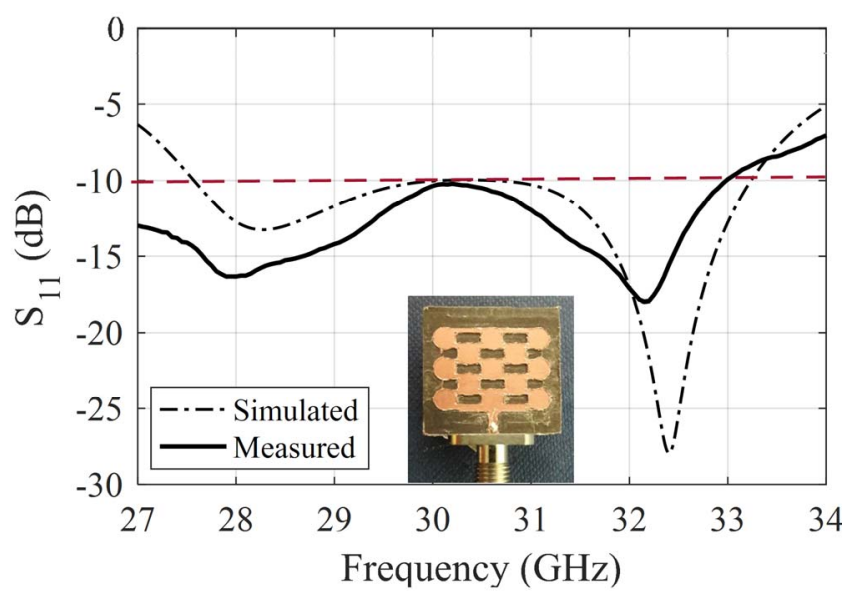

Fig. 3. Simulated and measured $S_{11}$ profile of the proposed compact grid antenna with an inset figure of the fabricated prototype.

TABLE I. REALIZED PEAK GAIN OF THE PROPOSED ANTENNA

\begin{tabular}{|c|l|l|l|l|l|l|l|}
\hline \multirow{2}{*}{$\begin{array}{c}\text { Gain } \\
\text { (dBi) }\end{array}$} & \multicolumn{7}{|c|}{ Frequency (GHz) } \\
\cline { 2 - 8 } & $\mathbf{2 7}$ & $\mathbf{2 8}$ & $\mathbf{2 9}$ & $\mathbf{3 0}$ & $\mathbf{3 1}$ & $\mathbf{3 2}$ & $\mathbf{3 3}$ \\
\hline $\begin{array}{l}\text { Sim. } \\
\text { Gain }\end{array}$ & 7.8 & 11.18 & 11.31 & 10.86 & 10.91 & 11.03 & 10.9 \\
\hline $\begin{array}{l}\text { Mea. } \\
\text { Gain }\end{array}$ & 8.9 & 11.02 & 11.57 & 11.55 & 11.12 & 9.45 & 6.16 \\
\hline
\end{tabular}


$(\mathbf{G H z})$

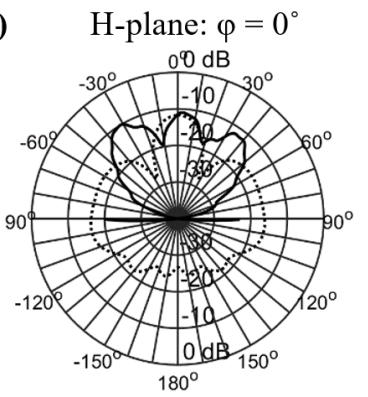

$00 \mathrm{~dB}$

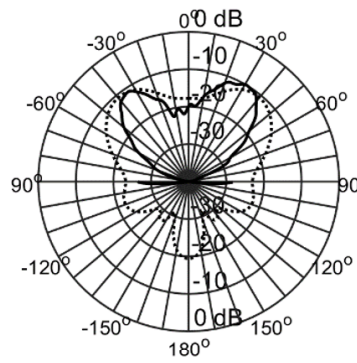

$00 \mathrm{~dB}$

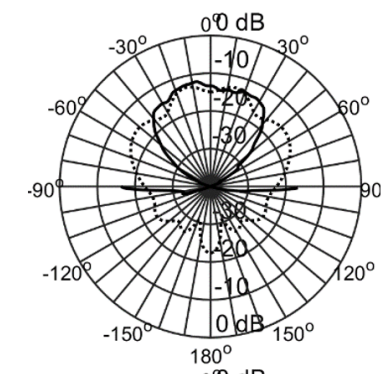

$00 \mathrm{~dB}$
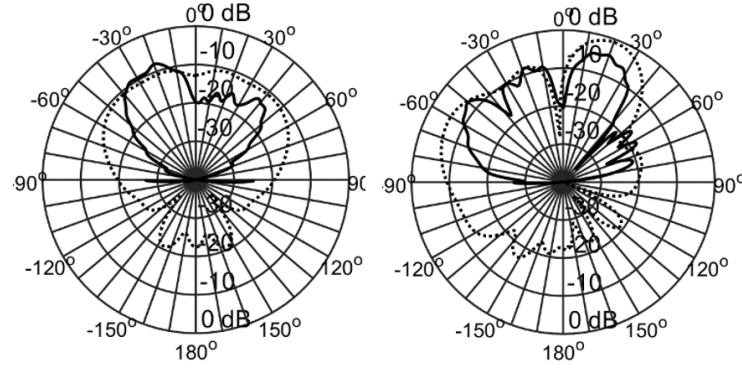

Fig. 4. Simulated and measured radiation pattern of the proposed compact grid antenna.

\section{B. 4-Element MIMO Array}

The simulated S-parameters plots of the designed MIMO antenna array are shown in Fig. 5 where all the four elements of the 2D MIMO array display same reflection coefficient response (in shown in Fig. 5(a)). It is essential to account for the mutual coupling between the individual antennas of the MIMO array to avoid interference from the neighbouring antennas. The simulated transmission characteristics of the MIMO antenna array in Fig. 5(b) shows low magnitudes below $-27 \mathrm{~dB}$ in the complete operating bandwidth that implies to lower mutual coupling and high isolation between the adjacent antenna elements.

To obtain an insight of the effects associated with the excitation of one antenna on the neighbouring antennas the surface current density of the proposed MIMO antenna has been computed in the CST simulation. The surface current distribution plots examine how independent antennas are in their individual performance and extent of parasitic coupling with adjacent radiators. Fig. 6 shows the current density plot when only one antenna of the MIMO array is excited at a time. The plot shows lower parasitic coupling of the single antenna with the adjacent elements.

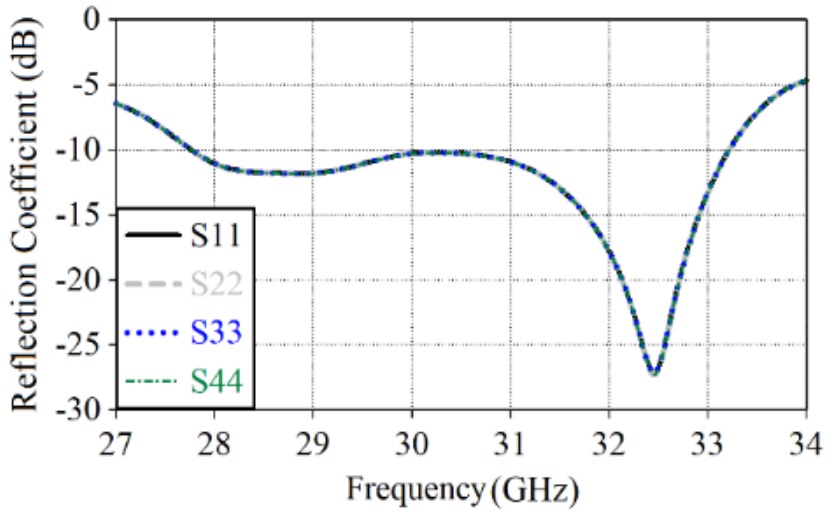

(a)

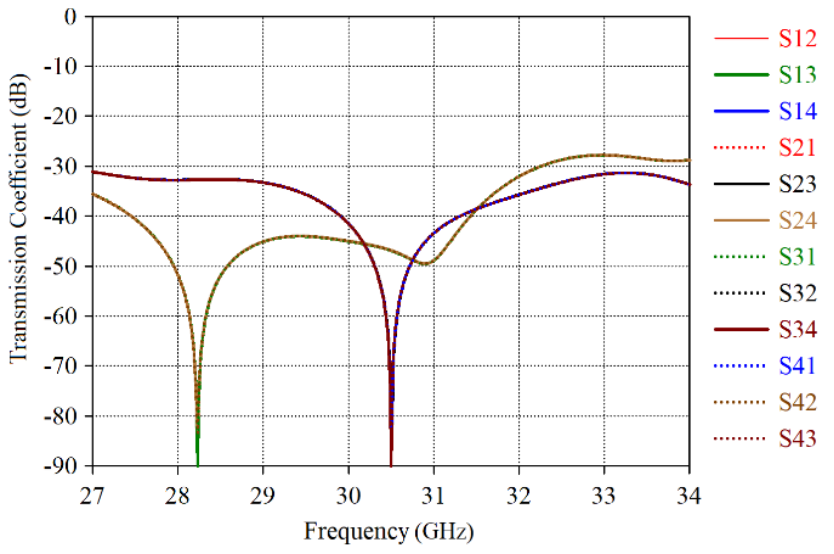

(b)

Fig. 5. Simulated S-parameters of the compact grid 4-element MIMO antenna array: (a) Reflection coefficient plots, (b) Transmission coefficient plots for the four antennas.

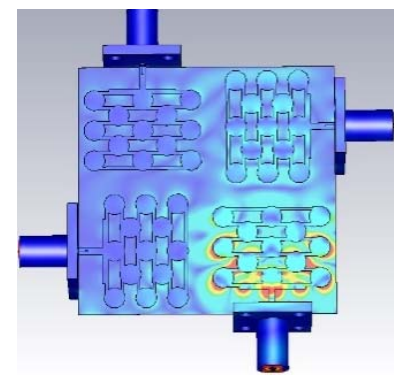

(a)

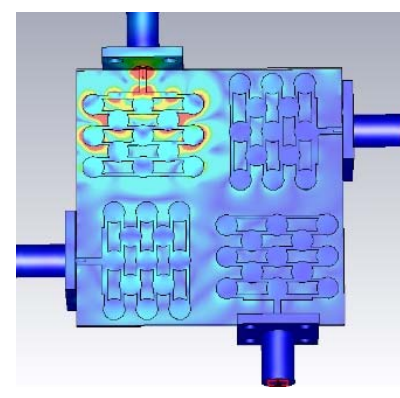

(c)

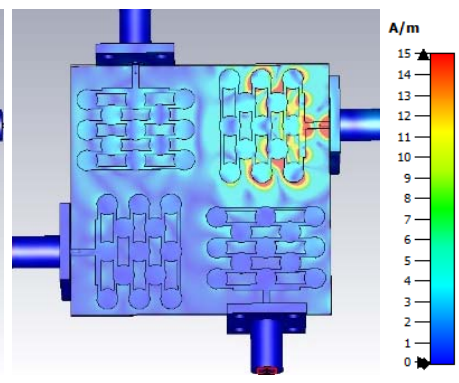

(b)

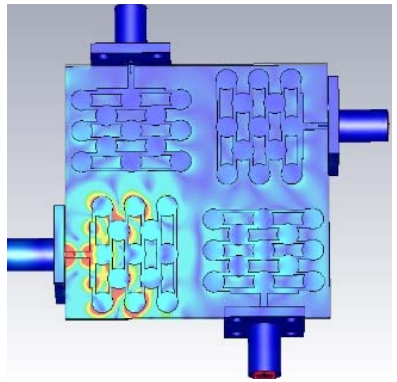

(d)
Fig. 6. Simulated surface current density profiles of the proposed compact grid 4-element MIMO antenna array: (a) Antenna I, (b) Antenna II, (c) Antenna III, (d) Antenna IV. 


\section{CONCLUSION}

This paper has presented a 4-element MIMO configuration of a compact microstrip-fed 2D grid array antennas as a potential solution for future wireless networks high gain and bandwidth demands are extremely critical. The designed geometry of a single grid antenna has thirteen circular patches each of the radius $0.175 \lambda_{0}$, arranged in a 2D planar assembly of a grid. The MIMO configuration is comprised of a compact orthogonal integration of four grid-array elements into an area of $42 \times 42 \mathrm{~mm}^{2}$.The measured results of the fabricated singleelement grid antenna show a bandwidth of $6 \mathrm{GHz}$ in the range of $27-33 \mathrm{GHz}$. The measured gain profile shows above $9 \mathrm{dBi}$ over almost the entire range with the peak gain of $11.75 \mathrm{dBi}$ at $29 \mathrm{GHz}$. Numerically estimated efficiency of the antenna is above $80 \%$. The 4-element MIMO shows lower mutual coupling with the transmission coefficients below $-27 \mathrm{~dB}$ in the overall bandwidth. These high-performance attributes suggest the suitability of the designed MIMO antenna for the advanced $5 \mathrm{G}$ networks and beyond.

\section{REFERENCES}

[1] D. Liu, W. Hong, T. S. Rappaport, C. Luxey and W. Hong, "What will $5 \mathrm{G}$ antennas and propagation be?," IEEE Trans. Antennas Propag., vol. 65, no. 12, pp. 6205-6212, 2017.

[2] W. Hong, K. H. Baek and S. Ko, "Millimeter-wave 5G antennas for smartphones: Overview and experimental demonstration," IEEE Trans. Antennas Propag., vol. 65, no. 12, pp. 6250-6261, 2017.

[3] A. I. Sulyman, A. Alwarafy, G. R. MacCartney, T. S. Rappaport and A. Alsanie, "Directional Radio Propagation Path Loss Models for Millimeter-Wave Wireless Networks in the 28-, 60-, and 73-GHz Bands," in IEEE Transactions on Wireless Communications, vol. 15, no. 10 , pp. 6939-6947, Oct. 2016.

[4] Use of Spectrum Bands Above $24 \mathrm{GHz}$ for Mobile Radio Services, Federal Register, vol. 81, no. 219, Nov. 2016.

[5] L. Wei, R. Q. Hu, Y. Qian, and G. Wu, "Key elements to enable millimeter wave communications for $5 \mathrm{G}$ wireless systems," IEEE Wireless Commun., vol. 21, no. 6, pp. 136-143, 2014.
[6] T. S. Rappaport, J. N. Murdock and F. Gutierrez, "State of the art in 60-GHz integrated circuits and systems for wireless communications," Proc. IEEE, vol. 99, no. 8, pp. 1390-1436, 2011.

[7] R. Rodriguez-Cano, S. Zhang, K. Zhao and G. F. Pedersen, "Reduction of Main Beam-Blockage in an Integrated 5G Array With a MetalFrame Antenna," in IEEE Transactions on Antennas and Propagation, vol. 67, no. 5, pp. 3161-3170, May 2019.

[8] S. F. Jilani and A. Alomainy, "Millimetre-wave T-shaped MIMO antenna with defected ground structures for $5 \mathrm{G}$ cellular networks," IET Microwaves, Antennas Propag., vol. 12, no. 5, pp. 672-677, 2018.

[9] S. Li, T. Chi, Y. Wang and H. Wang, "A millimeter-wave dual-feed square loop antenna for $5 \mathrm{G}$ communications," IEEE Trans. Antennas Propag., vol. 65, no. 12, pp. 6317-6328, 2017.

[10] H. A. Diawuo and Y. Jung, "Broadband proximity-coupled microstrip planar antenna array for 5G cellular applications," IEEE Antennas Wireless Propag. Lett., vol. 17, no. 7, pp. 1286-1290, 2018.

[11] S. F. Jilani et al., "A Ka-band antenna based on advanced Franklin array model for 5G cellular networks," Microwave and Optical Technology Letters, vol. 60, pp. 1562-1566, 2018.

[12] A. Dadgarpour, M. Sharifi Sorkherizi and A. A. Kishk, "High-efficient circularly polarized magnetoelectric dipole antenna for $5 \mathrm{G}$ applications using dual-polarized split-ring resonator lens," IEEE Trans. Antennas Propag., vol. 65, no. 8, pp. 4263-4267, 2017.

[13] S. F. Jilani and A. Alomainy, "A multiband millimeter-wave 2-D array based on enhanced Franklin antenna for 5G wireless systems," IEEE Antennas Wireless Propag. Lett., vol. 16, pp. 2983-2986, 2017.

[14] Z. Chen and Y. P. Zhang, "FR4 PCB grid array antenna for millimeterwave $5 \mathrm{G}$ mobile communications," IEEE MTT-S Int. Microw. Workshop Series on RF and Wireless Tech. for Biomed. Healthcare Applications (IMWS-BIO), 2013, pp. 1-3.

[15] S. F. Jilani, M. O. Munoz, Q. H. Abbasi and A. Alomainy, "MillimeterWave Liquid Crystal Polymer Based Conformal Antenna Array for 5G Applications," in IEEE Antennas and Wireless Propagation Letters, vol. 18, no. 1, pp. 84-88, Jan. 2019.

[16] S. F. Jilani, Q. H. Abassi and A. Alomainy, "Millimeter-wave compact and high-performance two-dimensional grid array for $5 \mathrm{G}$ applications,” 2019 IEEE Int. Symp. Antennas Propag. and USNCURSI Radio Science Meeting, 2019, pp. 25-26. 